\title{
Review of development of phase change cold storage technology for food cold chain application
}

\author{
Xiao-yan $\mathrm{Li}^{1, \mathrm{a}^{*}}$, Bing-qi Ma, ${ }^{2, b}$, Yue-ming $\mathrm{Li}^{3, \mathrm{c}}$ \\ ${ }^{1,2}$ School of Energy and Building Engineering, Harbin University of Commerce, \\ No. 1 Xuehai Street, Songbei District Harbin, Heilongjiang, 150028, China \\ ${ }^{3}$ Harbin Institute of Technology KINT Technology company, LTD \\ No. 92 West Street, Nangang District Harbin, Heilongjiang, 150000, China

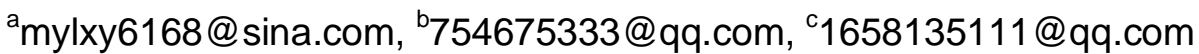

Keywords: Phase change cold storage technology; Refrigerated vehicle; Characteristic of charging; Numerical simulations.

Abstract. This paper reviews the recent development of phase change cold storage technology for food cold chain application. According to the application field of cold chain, developments of phase change cold storage technology in cold storage and low temperature transport are introduced separately, such as hold-over plate refrigerated storage, hold-over plate refrigerated vehicle, cold storage type transport incubators. Numerical simulations using the computational fluid dynamics (CFD) software ANSYS FLUENT to characterize the temperature distribution in cold storage plate and hold-over plate refrigerated vehicle in charging process is reported. The results indicate that the charging capacity increase with the heat transfer fluid flow rate increase when the inlet temperature remains constant. Application of phase change cold storage technology in field of refrigeration transportation equipment has huge development prospect.

\section{Introduction}

Food refrigeration freezing has been a major energy consumption industry because of its usage and wide range of application. It is necessary to study the energy-saving technology of the cold chain logistics, and make it sustainable development. The application of cold storage technology in the food cold chain energy-saving is a very good development direction [1].

Cold storage technology refers to the refrigeration unit starts to operate when the electricity load is low at night, the cold capacity produced is stored in a cold storage medium, and released at the peak of power load to meet the demand of peak cooling load or cold in the production process, and eventually achieve the purpose of peak load shifting and power saving.

Phase change thermal storage technology is suitable for food cold chain, with the principle of phase change thermal energy storage, the materials of different phase change temperature can be configured reasonably to achieve effect of phase change thermal storage according to the different temperature requirements of the cold chain transportation. Such as cold storage type transport incubator, beer ice bags produced by using phase change cold storage technology, which are used for cooling and refrigeration in the process of transportation and sale of beer, the purpose is to ensure fresh and cool taste of the beer. Therefore, the phase change cold storage technology has broad application prospects and energy-saving potential in every link of food cold chain low temperature food processing, cold storage, cold chain transportation and distribution, cool temperature sales $[2,3,4]$. Ninety-eight percent of Japanese fruits and vegetables use the cold chain in the circulation transportation [5].

\section{Application of phase change cold storage technology in cold storage}

Ice cold storage and cold storage technology for vegetables and fruits preservation. Ice cold storage and cold storage technology for vegetables and fruits preservation is a new technology based on the cold storage technology to keep vegetables and fruit fresh. In the 1970s, many scholars from the United States, Britain, Canada and other countries also have a wide range of research on 
this project, and there is fresh cold storage using ice cold storage and cooled wet air for flowers, vegetables and fruit preservation in foreign countries. LiyuanWu, Kangning Guo, Wen Su [6,7,8] etc, have also put forward to cool the condensed water down for cold storage at night to lower the condensation temperature, and they have basically completed the theoretical and experimental model of wet cooling system.

Hold-over plate refrigerated storage. Hold-over plate refrigerated storage is similar to refrigerated vehicles. The cold storage board is installed under its ceiling or in both sides of the body. The artificial cooling capacity is stored by the sensible heat or latent heat of the material and used during the peak of load. Fig. 1 and Fig. 2 respectively show top-mounted and side-mounted installation methods of the cold storage plate and refrigeration pipe [6].

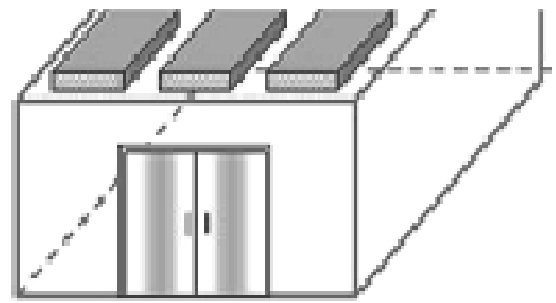

Fig. 1 Top-mounted storage plate

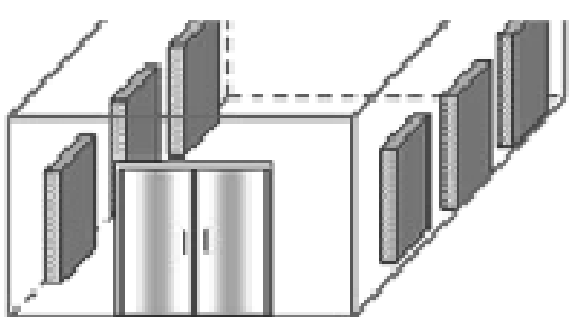

Fig. 2 Side-mounted storage plate

\section{Application of phase change cold storage technology in low temperature transport}

Our country has the huge potential for the cold chain transportation market, but the cold chain logistics foundation is weak, it is difficult to narrow the gap with the developed countries in a period of time [9]. After the 1960s, cold storage technology has already been widely used in refrigerator vehicles and refrigerator containers. Storage panels filled with eutectic solution as the cold source are installed on heat insulated body. At present, China has developed the second generation storage plate refrigerator vehicles (mechanical plate refrigerator vehicles, including rail and highway) [10], which have overcome the disadvantage of charging rechargeable cold only on the ground stations of the first generation cold plate refrigerator vehicles. The refrigeration unit is taken with the refrigerator vehicles. The cold charging could be taken place everywhere as long as the supply of electricity, making it possible to be widely used.

From the classification of cooling method, refrigerated transport can be divided into ice refrigeration, mechanical refrigeration, refrigerated containers and hold-over plate refrigerated vehicle $[11,12,13]$. Refrigerated containers and hold-over plate refrigerated vehicles are the key to the development. Hold-over plate refrigerated vehicle have a good effect in energy-saving, such as China first generation cold plate refrigerated railroad car, which has run 12 times and the average transport distance is $2120 \mathrm{~km}$ from September in 1983 to August in 1984.

Hold-over plate refrigerated vehicle. The compartment system of the hold-over plate refrigerated vehicle is made of foam molding with rigid polyurethane, which can reduce the loss of cold. Cold storage plates are generally symmetrically set on both sides or the top of the envelope with the thickness from $50 \mathrm{~mm}$ to $100 \mathrm{~mm}$, and the hollow body is made of aluminum alloy with coolant injected. Fig. 3 shows the structure of the cold storage plate [14].

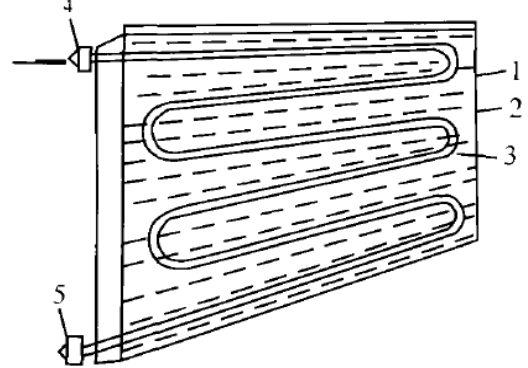

Fig. 3 The structure of the cold storage plate 1. Cold storage plate 2. Cold storage liquid

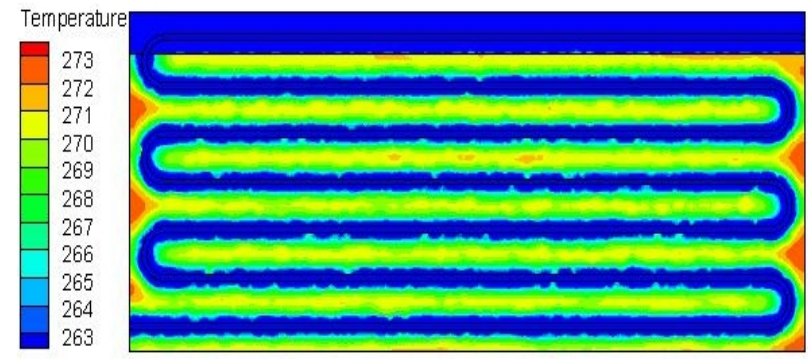

Fig. 4 Temperature distribution of cold storage plate 
3. Heat exchange coil 4. Inlet of refrigerant

5. Outlet of refrigerant

Li [15] presented a mathematical model that uses the ANSYS method to describe the storage characteristics of cool storage medium in the hold-over plate, analyzed the influence of inlet flow rate and inlet temperature on the outlet temperature and the charging capacity. Fig. 4 is the temperature distribution of cold storage plate in process of cold storage at the end of 3600 seconds. .

Liu [16] provided a numerical simulation method to simulate the distribution of the cold storage plate refrigerated vehicles temperature field simulation of temperature field, it's shown in Fig. 5. The results show that: the temperature of its compartments suitable for refrigerated trucks refrigerated transport.

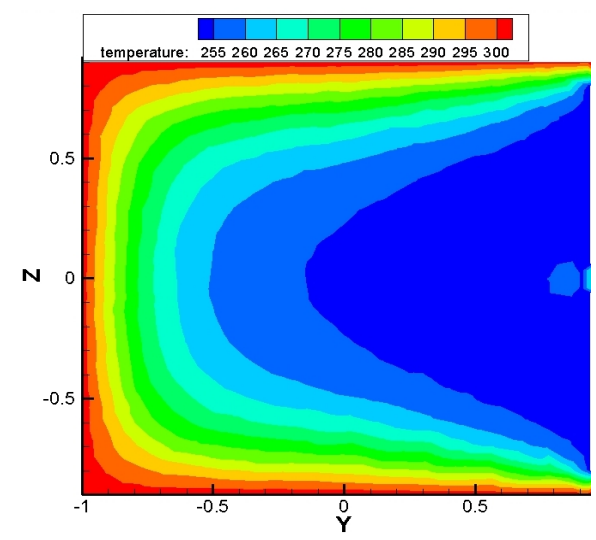

Fig. 5 Temperature distribution of the cold storage plate refrigerated vehicles

Ming Liu [17] investigated a kind of new cold storage type refrigerator vehicle, which has lower noise, lower power, and more suitable for small and medium-sized refrigerator vehicles for short-haul and local transportation compared with conventional systems. Mashud Ahmed [18] from America has studied on insulation material of the carriage of the vehicle. He found the insulation material directly affects heat-insulating property, mechanical strength, environmental performance and economy.

Cold storage type transport incubators. Cold storage type transport incubator is a kind of effective logistics technology and equipment [19]. It is a high quality transportation equipment of small amount, high quality goods, which has the characteristics of central cooling, high capacity utilization of the volume, light weight, high working efficiency, and fast loading. The main structure of the incubator is storage tank and the heat plates. The incubators have the function of cooling. The temperature is controlled with cold storage plates with different temperatures, and the cold can be kept for a long time without refrigeration cold. Efficient coolant has been injected into the cold storage panels and can be used for several times. According to the number and type of cold storage plate, the control of different temperature depends on different specifications of cold storage plates. Cold storage plate released the cold to keep the temperature stable in the incubator. Frozen goods and refrigerated goods were kept in different temperature incubator, and then they can be loaded in the common vehicles as the goods for delivery. Compared with the traditional refrigerator vehicles, cold storage type transport incubator is flexibility, environmental, economic, safety, controllability [20].

\section{Challenges and technology perspective}

Technology perspectives with regard to phase change cold storage technology for cold chain are mainly summarized as follows:

1. Develop cold storage materials or storage systems with controllable temperature.

2. Research on the relationship between the structure of phase change material and the latent heat and find best ways to improve the latent heat of phase change materials.

3. Research on the cold storage mechanism of the cold chain cold storage medium to improve cold storage and release characteristics of phase change materials to reduce the charging time of the cold storage plate. 
4. Theoretical analysis and experimental on the improvement of the characteristics of the cold storage plate. Optimize the design of cold storage plate refrigerated vehicle and reform the operation and management in the current system.

5. Study on new portable cold storage insulation boxes.

6. Research on new cold storage and refrigerated storage cabinets.

7. Economic Analysis and Evaluation of the cold chain cold storage technology.

\section{Acknowledgments}

The authors would like to acknowledge the project (51476049) supported by National Natural Science Foundation of China.

\section{References}

[1] Yuguo Zhao, J. Contemporary Economics, 9 (2008) 67. In Chinese

[2] Xiaoyan Li, Kaidi Li, Jiaqing Liu, Research and progress of phase change storage technology application in the food cold chain, C. Wuhan, 2013. In Chinese

Reference to a book: 2013 China Refrigeration Institute Academic Essays

[3] Yuan Chang, Zeqin Liu, The preliminary discussion on the development of refrigerated transport and refrigerated transport equipment, C. Qingdao, 9 (2010) 164-167. In Chinese

Reference to a book: Proceedings of the 7th National Conference on Food Cold Chain

[4] Zhiqiang Zhu, Xiaoshuan Zhang, J. China Fruit Vegetable, 34 (2014) 14-18. In Chinese

[5] Jiang Shen, Bin Liu, J. Journal of Refrigeration, 30 (2009) 20-25. In Chinese

[6] Liyuan Wu, Wenji Song, Rixin Gao, J. Journal of Refrigeration, 33 (2012) 66-69. In Chinese

[7] Kangning Guo, J. Refrigeration, 2 (1997) 56-60. In Chinese

[8] Wen Su, Rudong Chen, J. Refrigeration Technology, 4 (2002) 31-35. In Chinese

[9 ] Jing Xie, J. Refrigeration Technology, 3 (2010) 5-10. In Chinese

[10] Liu Guofeng, Xie Chen. Dongren, Shi Lei, J. Railway vehicle, 10 (1998) 33-34. In Chinese

[11] Guofeng Liu, Zhongzhi OuYang, J. Refrigeration, 26 (2007) 27-30. In Chinese

[12] S. A. Tassou, G. De-Lille, Y. T. Ge, J. Applied Thermal Engineering, 29 (2009) 1467-1477.

[13] S. J. James, C. James, J. A. Evans., J. International Journal of Refrigeration, 29 (2006) 947-957.

[14] Yingui Fang, J. Refrigeration technology, 4 (1998) 23-26. In Chinese

[15] Yueming Li, Study on basic of application of cool storage materials for refrigerated transport vehicle, D. (2015). In Chinese

[16] Jiaqing Liu, Study on the cool storage material used for cool storage refrigerated vehicles and its thermal performance, D. (2014). In Chinese

[17] Ming Liu, Wasim Saman, J. Applied Energy, 92 (2012) 336-342.

[18] Mashud Ahmed, Oliver Meade, J. Energy Conversion and Management, 51 (2010) 383-392.

[19] Fan Hu, J. Logistics Equipment, 4 (2008) 35. In Chinese

[20] Hui Fang, J. Food Safety Guide, 4 (2009) 86-87. In Chinese 